Missed Diagnosis

\title{
Leaking thoraco-abdominal aortic aneurysm presenting with a massive left haemothorax: a clinical trap
}

\author{
Simon P.J. Huddy, William P. Joyce and John R. Pepper
}

South West Thames Regional Cardiothoracic Unit, St George's Hospital, Blackshaw Road, London SWI7 $O Q T, U K$.

\begin{abstract}
Summary: A case is described in which a patient, who had had an aortic aneurysm repair 10 years previously, presented with a thoraco-abdominal aortic aneurysm which ruptured immediately above the previous graft. The implications for the management of patients with an abdominal aortic aneurysm repair are discussed.
\end{abstract}

\section{Introduction}

A leaking thoracic aneurysm typically presents with chest pain, widening of the mediastinum on chest X-ray and a left haemothorax. In this report we present a thoraco-abdominal aneurysm which leaked from the abdominal portion but which presented with a massive left haemothorax leading to inappropriate surgery.

\section{Case report}

A 78 year old man presented with a 24-hour history of left hypochondrial, left chest and high central back pain of sudden onset. Ten years previously he had undergone elective replacement of an infrarenal abdominal aortic aneurysm with a dacron graft. At this time the suprarenal aorta appeared not to be dilated and a chest X-ray was reported as normal. He remained well from the time of operation until the present admission.

On examination at the time of admission he was in pain, pale and clammy, pulse rate $90 / \mathrm{min}$, blood pressure $210 / 110 \mathrm{mmHg}$. A brief period of hypotension prior to transfer had responded to infusion of 2 litres of colloid solution. There were the physical signs of a left pleural effusion, mild left upper abdominal tenderness and the abdominal aorta was palpable.

Chest X-ray revealed a widened thoracic aorta with a large left pleural effusion. A previous film taken 12 hours earlier at the referring hospital did

Correspondence: S.P.J. Huddy, M.Chir, F.R.C.S., Royal Surrey County Hospital, Park Barn, Guildford, Surrey GU2 5XX, UK.

Accepted: 9 September 1989 not show the effusion. Abdominal ultrasound demonstrated an aneurysmal upper abdominal aorta tapering to a normal calibre at the level of the previous graft. A computed tomographic (CT) scan performed with intra-arterial contrast showed the aorta to be dilated from the level of the carina to the origin of the renal vessels. There was a suspicion of a leak of contrast at the level of T6. The pleural effusion was again noted (Figure 1). An aortogram failed to demonstrate the leak.

The aorta was explored through the left chest which was found to contain a large quantity of fresh blood clot. The aorta was aneurysmal and there was extensive haematoma within the mediastinum. The aorta was mobilized proximally and one end of a 'Gott' shunt inserted. This is a polyvinyl tube, tapered at each end, which has heparin bonded into the wall. It allows a bypass of the affected aorta without systemic heparinisation.' At this point there was evidence of a sudden loss of circulating volume although only a relatively small quantity of blood was seen in the chest. Attempts at resuscitation were unsuccessful and the patient died.

A post-mortem examination was performed which demonstrated an aneurysmal aorta from the origin of the left subclavian artery to the previous graft in the abdomen. There was free blood within the abdominal cavity and a large tear immediately above the origin of the previous graft. It was clear that this was the site of bleeding. A haematoma within the upper retroperitoneum extended into the mediastinum.

\section{Discussion}

As an increasing number of patients now undergo successful replacement of the abdominal aorta for 


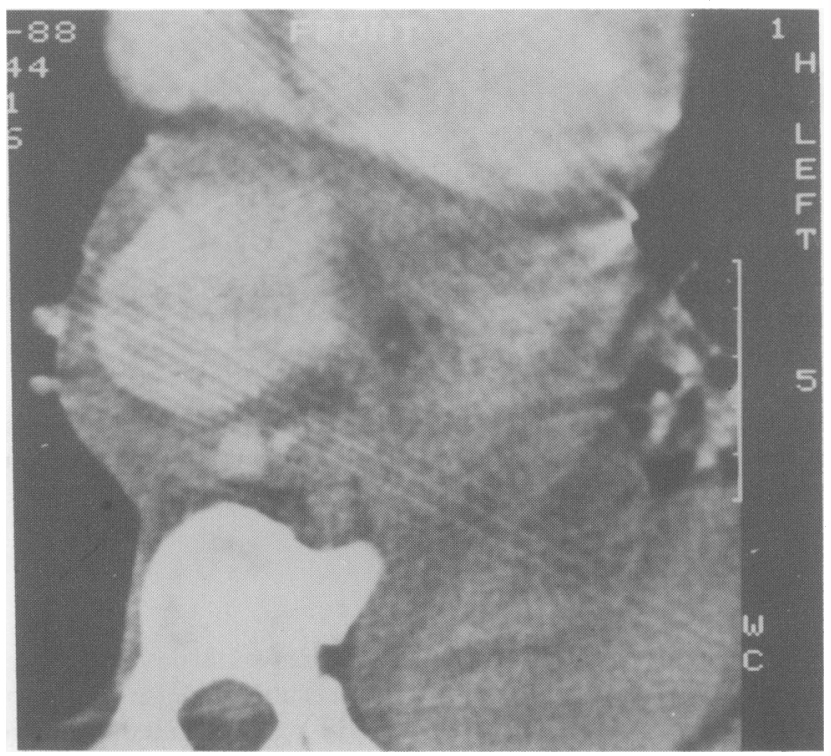

Figure 1 CT scan at the level of the sixth thoracic vertebra after intra-arterial contrast which shows an aneurysmal aorta with blood in the left chest.

an aneurysm. Crawford and Cohen have demonstrated that $12 \%$ of patients with this condition have further aneurysms in other sites when these are searched for. ${ }^{2}$ The incidence of detected second aneurysms appears to be increased by modern investigation techniques such as CT scanning. However, in our case the suprarenal aorta and chest X-ray were normal at the time of the first operation but subsequently became dilated. A case has recently been reported in which the infrarenal aorta above an aortic graft, inserted at aneurysm repair, subsequently became dilated. ${ }^{3}$ These cases serve to remind us that the disease process is a generalized one and continues after correction of the initial abnormality. Thus follow-up of patients who have undergone replacement of the abdominal aorta should include a search for further aneurysms. Appropriate treatment of these, when detected, may prevent rupture with the associated poor prognosis. Crawford and Cohen ${ }^{2}$ suggested that this should be by an annual chest X-ray and abdominal ultrasound examination.

The presentation of our case with a haemothorax is unusual and was misleading as to the site of rupture. A massive left haemothorax due to rupture of an abdominal aortic aneurysm has been previously reported in a post-mortem series of patients who died without operation ${ }^{4}$ and a right sided haemothorax has been reported in a woman who presented with a simultaneous perforated duodenal ulcer and leaking abdominal aortic aneurysm. ${ }^{5}$ In our case it seems likely that fibrosis surrounding the previous aortic graft obliterated the potential retroperitoneal space and that this caused the haematoma to track cranially, into the mediastinum, and eventually rupture into the pleural cavity. The history of upper abdominal and high back pain, together with a left haemothorax was suggestive of an intrathoracic leak. The CT scan and aortogram both clearly demonstrated the presence of aneurysmal dilatation of the aorta from the arch extending down into the abdomen, but did not demonstrate the site of leakage. The presence of a massive left haemothorax led to the conclusion that the bleed was within the chest although this was subsequently found not to be the case.

\section{References}

1. Gott, V.L. Heparinized shunts for thoracic vascular operations. In: Saliston, D.C. and Spencer, F.C. (eds) Gibbon's Surgery of the Chest. W.B. Saunders and Co., Philadelphia, 1983, pp. 976-987.

2. Crawford, S. \& Cohen, E.S. Aortic aneurysm: A multifocal disease. Arch Surg 1987, 117: 1393-1400.

3. Millard, F.C. \& Powis, S.J.A. A case of metachronus aneurysm of the infrarenal abdominal aorta. Postgrad Med J 1988, 64: 403-404.

4. Darling, R.C. Ruptured arteriosclerotic abdominal aortic aneurysms: A pathologic and clinical study. Am J Surg 1970, 119: 397-401.

5. Karam, P.G., Novick, R.J., Glickman, L.T. \& Blundell, P.E. Right haemothorax: an unusual presentation of ruptured infrarenal abdominal aortic aneurysm. Can J Surg 1986, 29: $133-134$. 